\title{
Ovulation induction in polycystic ovarian syndrome - basics for gynecologist
}

\author{
Punita Yadav* \\ Department of Obstetrics and Gyanecology, B. P. Koirala Institute of Health Sciences, Dharan, Nepal
}

Received: 28 December 2020

Accepted: 05 February 2021

\section{*Correspondence:}

Dr. Punita Yadav,

E-mail: punita_yadav@hotmail.com

Copyright: $(\odot$ the author(s), publisher and licensee Medip Academy. This is an open-access article distributed under the terms of the Creative Commons Attribution Non-Commercial License, which permits unrestricted non-commercial use, distribution, and reproduction in any medium, provided the original work is properly cited.

\begin{abstract}
Polycystic ovarian syndrome (PCOS) is the most common problem related to infertility among women. This review looked into the evidence and options available for ovulation induction. The management protocol and their respective efficacy was looked into during the write up of the review. Weight reduction, along with metformin therapy are the initial and efficient way to regain ovulation among obese female. This should be complemented by use of first line medicine like aromatase inhibitors and selective estrogen receptor modulators. The first line is effective in significant proportion of female to induce ovulation and increase live birth rate. However, for those not responding should initially undergo the use of gonadotropins and ovarian drilling. Finally, controlled ovarian stimulation and in vitro fertilization should be considered as it is an invasive, expensive and there are risks of ovarian hyperstimulation and multiple gestation.
\end{abstract}

Keywords: PCOS, Ovulation induction, Gonadotropins, Live birth

\section{INTRODUCTION}

Polycystic ovary syndrome (PCOS) is a heterogenous disorder affecting women of adolescent age, child bearing age group and also postmenopausal age. It is an important cause of ovulatory dysfunction, menstrual irregularity and androgen excess. It affects about $20 \%$ of the adolescent and adult female in Indian subcontinent. ${ }^{1,2}$ PCOS is one of the most common cause of anovulation and infertility, it accounts to about 25 to $30 \%$ of the cause of infertility in women. ${ }^{3}$

The relationship between hyperandrogenism and anovulation is complex. In PCOS women there is an increased pulsatility of gonadotropin-releasing hormone $(\mathrm{GnRH})$, resulting in increased release of luteinizing hormone (LH) and an elevated LH/follicle-stimulating hormone (FSH) ratio. ${ }^{4}$ Because of low level of FSH level, follicles growth is arrested at different stage of maturation (2-10 $\mathrm{mm}$ diameter). This results in decreased estrogen and increased inhibin production. Elevated LH causes more androgen production from theca cells or from stroma. FSH induces aromatization of this androgen to estrogen, resulting in androgenic follicular microenvironment. This all process ultimately results in anovulation. ${ }^{5}$ Several methods are used for effective ovulation which includes lifestyle modification directed towards weight loss, clomiphene citrate, metformin, gonadotropins, ovarian drilling, invitro fertilization, etc. This review will discuss on the treatment modalities available for induction in PCOS.

\section{METHODOLOGY}

A literature searches in PubMed, Google Scholar and Cochrane Database using the keywords and MeSH term polycystic ovarian syndrome, ovulation, infertility, obesity, insulin sensitizer, aromatase inhibitor, letrozole, metformin, gonadotropins, ovarian drilling, in vitro fertilization, estrogen receptor modulators was done. Important and relevant information was extracted to give completeness to this review article. 


\section{DISCUSSION}

There are few important steps to be undertaken before initiating ovulation induction, which includes history and evaluation of both the couple. Specifically, history regarding regular sexual intercourse, use of any contraceptive measures, discussion on the expenditure of the therapy, evaluation of the couple stress and state of mind, their availability of time and their dedication towards therapy and regular follow up should be done. Similarly, evaluation of tubal patency by hysperosalpingography (HSG) or laparoscopy with chromotubation along with semen analysis are mandatory before starting the medical therapy. ${ }^{6}$ Different modalities of ovulation induction in PCOS will be discussed in a sequential manner.

\section{Weight reduction and life style modification}

Weight reduction along with lifestyle modification should be the first step in the management of overweight PCOS, because this has been proven to be effective in restoring ovulatory cycles and achieving pregnancy. This has also shown to improve the efficacy of medical therapies and improve the likelihood of pregnancy with live birth. Preconception lifestyle modification, like cutting of calories with meal replacements, exercise, use of oral weight loss medication like orlistat or sibutramine has shown to increase the ovulatory rate. After weight reduction of $7 \%$ in a clomiphene induced cycle, cumulative ovulation could rise by $60 \%$, with rise in live birth rates by $26 \% .^{7}$ In addition six weeks of structured exercise training and hypocaloric diet would increase the likely hood of ovulation with clomiphene in clomiphene resistant overweight PCOS by a factor of $4 .^{8}$

Regular exercise and weight loss increase insulin sensitivity and improves metabolic parameters. Excessive caffeine intake, alcohol consumption, and smoking should also be discouraged. ${ }^{9}$ Maintaining weight and diet also decrease maternal complications like gestational hypertension, gestational diabetes mellitus, thromboembolism, and wound infection. ${ }^{10}$ Lifestyle modifications is also a modifiable risk factor for long term cardiovascular disease and type 2 diabetes among women with PCOS.

\section{Insulin sensitizing agents (metformin)}

PCOS is a state of insulin resistance and hyperinsulinemia. ${ }^{11}$ Metformin is a biguanide and it acts by inhibiting hepatic glucose production and increasing peripheral glucose intake. Its role as an insulin sensitizer has led to its widespread use in PCOS in conjunction with other ovulation inducing agents. ${ }^{12}$ However, there are very limited use of other insulin sensitizing agents like Dchiroinositol, rosiglitazone or pioglitazone, and are not discussed here. Metformin alone could significantly increase the ovulation rate vs placebo (23\% vs $13 \%$ ), while it does not significantly increase the pregnancy or live birth rate. ${ }^{13,14}$ Similarly, a recent Cochrane review suggests that metformin may improve live birth rates compared with placebo (odds ratio (OR) 1.59 , 95\% CI 1.00 to 2.51 ). Although, there were more gastrointestinal side effects (OR 4.76, 95\% CI 3.06 to 7.41), metformin had higher rates of clinical pregnancy (OR 1.93, 95\% CI 1.42 to 2.64 ), ovulation (OR 2.55 , $95 \%$ CI 1.81 to 3.59 ) and improved menstrual frequency (OR 1.72, 95\% CI 1.14 to 2.61). There was no clear evidence of a difference in miscarriage rates (OR $1.08,95 \%$ CI 0.50 to 2.35). ${ }^{15}$ Metformin plus clomiphene citrate had higher rate of clinical pregnancy then clomiphene citrate alone (OR $1.59,95 \%$ CI 1.27 to 1.99 ) and ovulation was also higher (OR $1.57,95 \%$ CI 1.28 to 1.92 ). The anticipated clinical pregnancy rate and live birth rate could be projected to 338 vs 243 and 295 vs 257 per 1000 women.

Hence, it is advisable to use metformin and clomiphene combined irrespective of obesity status among PCOS women. ${ }^{15}$ The advised starting dosage of metformin should be $500 \mathrm{mg}$ daily with main meal, for 1-2 weeks which should be increased weekly or biweekly by 500 $\mathrm{mg}$ a day until a maximum dose of 2000-2500 $\mathrm{mg} /$ day is reached depending on the clinical benefit and side effects. If the dose increase results in worsening of the side effects, the current dose can be maintained for 2-4 weeks until tolerated. To lessen the side effects slow-release metformin can be used. ${ }^{16}$

\section{Selective estrogen receptor modulators-clomiphene citrate}

Clomiphene acts by binding to estrogen receptors and thus has an antiestrogenic effect both on hypothalamus and pituitary. This results in increase in FSH which in turn causes follicular development, increase in estradiol is followed by endogenous LH surge. High FSH level in early follicular phase may result in recruitment of more than one follicle. Clomiphene is an effective first line medicine for ovulation induction, however there are risks of multiple pregnancy. ${ }^{17}$

Compared to placebo clomiphene can increase the pregnancy rate (OR 5.8, 95\% CI:1.6-21.5), with up to an $11 \%$ risk of multiple pregnancy. ${ }^{18,19}$ Hence, regular USG to evaluate the ovarian response should be monitored. The starting dose of clomiphene is $50 \mathrm{mg}$ /day from the 2nd day of the menstrual period and should be given for 5 days, the dose should be increased to $100 \mathrm{mg}$ /day if there is no ovulation for 2 cycles with the previous doses. Despite of a dosage of $100 \mathrm{mg} /$ day if the cycles remain anovulatory, the cases is levelled as clomiphene resistant and discontinuation of clomiphene therapy should be considered ${ }^{20}$ Following, clomiphene therapy 60 to $85 \%$ of PCOS undergoes ovulation, while only $50 \%$ of them conceives. ${ }^{21,22}$ The thinning of endometrium secondary to antiestrogenic usually prevents conception despite ovulation. Alternatives for ovulation induction should be considered if periovulatory endometrium remains persistently thin. Along with multiple gestations, 
vasomotor hot flushes, blurring of vision and increased risk of borderline ovarian tumor are potential side effects with clomiphene citrate..$^{23,24}$

\section{Aromatase inhibitors - letrozole}

AIs inhibits the cytochrome P450 isoenzymes $2 \mathrm{~A} 6$ and $2 \mathrm{C} 19$ of the aromatase enzyme complex and downregulate the production of estrogen. ${ }^{25}$ The negative feedback loop of oestrogen in the hypothalamus is inhibited, resulting in stronger gonadotropin-releasing hormone $(\mathrm{GnRH})$ pulses. This in turn stimulates the pituitary gland to produce more $\mathrm{FSH}$, which induces development of follicles in the ovaries. In contrast to Clomiphene, the central feedback mechanism remains intact and as the dominant follicle grows and oestrogen levels rise, normal negative feedback occurs centrally suppressing FSH and hence usually resulting in single dominant follicle. ${ }^{26}$

In a Cochrane database review, letrozole compared to clomiphene citrate (CC) with or without adjuncts has higher birth rate (OR 1.68, 95\% CI 1.42 to 1.99). However ovarian hyperstimulation syndrome rates are similar with letrozole or clomiphene citrate. There is evidence for a higher pregnancy rate in favour of letrozole (OR 1.56, 95\% CI 1.37 to 1.78 ). There is little difference between letrozole groups in the rate of miscarriage by pregnancy (19\% versus $20 \%$, OR 0.94 , $95 \%$ CI 0.70 to 1.26$)$ and multiple pregnancy rate $(1.3 \%$ versus $1.7 \%$; OR $0.69,95 \% \mathrm{CI}=0.41$ to 1.16$).{ }^{27}$

Letrozole is usually administered from the 3rd day of the menstrual cycle for total of 5 days. To start with dosage is usually begun at $2.5 \mathrm{mg}$ daily and the basis of development of adequacy of follicular size and endometrial thickness, as deemed necessary the dose could be increased to $5 \mathrm{mg}$ and subsequently to $7.5 \mathrm{mg}$ daily in the consecutive cycle on the basis of individual response. ${ }^{28}$ Letrozole as a side effects could cause fatigue and dizziness. But importantly concerns on congenital anomalies in fetus with letrozole therapy were raised. However, in recent studies it has been found that there are no significant differences in congenital anomalies between letrozole or clomiphene groups. ${ }^{29,30}$ Conclusively, letrozole could safely be used as a second line therapy for ovulation among PCOS women.

\section{Gonadotropins}

Gonadotropins are second line therapy for those who have failed to respond to Clomiphene therapy. This method of inducing ovulation is based on the concept that administration of FSH will results in follicular growth and development and FSH above threshold level for sufficient duration would generate a limited number of developing follicles. There are different approaches towards administration of gonadotropins. The preferred one is the step-up protocol. The FSH dosage is started at 37.5-75 IU/day from, if follicle development is not observed on USG after a week, dose is increased by $50 \%$. Once follicular growth is observed same dose is maintained until follicular selection is achieved. ${ }^{31}$ In stepdown protocol the higher dose 150 IU/day is given until there is follicular development in USG and subsequent stepwise reduction is done. ${ }^{32}$ However, step up is safer and easily instrumented in comparison to the later protocol. ${ }^{33}$ The consensus on fertility related to PCOS has also recommended the use of gonadotropins at initial dose of 37.5-50 IU/day to reduce the risk of ovarian hyperstimulation syndrome (OHSS). ${ }^{34}$ The low dose regimen has a good efficacy with ovulation rate of $70 \%$, pregnancy rate of $20 \%$ and multiple live birth rate of $<6 \%$ and OHSS rate of $<1 \% .{ }^{33,35}$ Short protocol with $\mathrm{GnRH}$ antagonist reduces the risk of hyperstimulation but also it interferes with LH surge and hence result in lower rate of pregnancies. ${ }^{36}$ It is also demonstrated that combine use of metformin along with FSH therapy elicit a lower rate of OHSS. ${ }^{37}$ Gonadotropins is a complex and expensive method of ovulation induction; it requires a tight monitoring along with risk associated with ovarian hyperstimulation and multiple gestation. Hence, this approach should be reserved as a last resort before proceeding towards invitro fertilization (IVF) technique.

\section{Laparoscopic ovarian drilling (LOD)}

Laparoscopic ovarian drilling is an invasive procedure reserved for its use when there is poor response of ovulation with medical management in women with PCOS. In this procedure by using laparoscopic ovarian cautery or diathermy multiple perforations are done in the ovarian surface and stroma. This destroy ovarian androgen-producing tissue and reduce the peripheral conversion of androgens to estrogen, which results in fall of LH and rise of FSH and increases the chances of ovulation. $^{38}$ LOD could increase the ovulation rate of ovulation induction therapy from $39 \%$ to $77 \%$ and pregnancy rate from $33 \%$ to $47 \% .^{39}$ Recent Cochrane review data suggests that LOD have a decreased live birth when compared to medical ovulation alone (OR $0.71,95 \%$ CI 0.54 to 0.92 ).

The evidence suggests that chance of medical ovulation alone is $42 \%$ while LOD could have ovulation rate between $28 \%$ and $40 \%$. Medical ovulation would have a risk of multiple pregnancy of 5\%, LOD decreases the risk of multiple pregnancy with medical ovulation between $0.9 \%$ and $3.4 \%$. However, there was little or no difference with miscarriage frequency. LOD however may reduce OHSS (OR 0.25, 95\% CI 0.07-0.91). There are limited study to suggest need of bilateral LOD versus unilateral LOD. ${ }^{40}$ Hence, LOD is an invasive option that could be provided to the women who are resistant to first line therapy of medical ovulation.

\section{In vitro fertilization}

IVF is an invasive option left for PCOS with failure of gonadotropin therapy or in combination with tube related 
issues of infertility, or have significantly reduced number or quality of sperms. In IVF, for multi-follicular development firstly, gonadotropins are administered after which oocyte retrieval is carried out, once embryos are generated, they are transferred to the uterus. Pregnancy rate can approach $40 \%$ to $50 \%$ per cycle with IVF. ${ }^{41}$ However, the risk of OHSS is significantly increased, even with the use of GnRH antagonist protocols. Time and cost related issues should always be discussed with the couple opting for IVF before enrolling for the therapy.

\section{CONCLUSION}

PCOS is the common problem in women of reproductive age group related to infertility. However, many timetested options are available. Base on the availability, individual metabolic status, complexity and adverse effects of treatment options, preferences should be made. All overweight women should undergo weight reduction protocol; metformin use has shown to be beneficial even when opting for inducing ovulation with other available options. First line for ovulation induction is either letrozole or clomiphene citrate. For those resistant to first line therapy gonadotropins along with ovarian drilling could be considered. Finally, for those not ovulating or conceiving or having issues related to fallopian tubes or semen should undergo IVF.

\section{ACKNOWLEDGMENTS}

Dr. Shankar Prasad Yadav for helping in literature search and synthesis of the review.

\section{Funding: No funding sources}

Conflict of interest: None declared

Ethical approval: Not required

\section{REFERENCES}

1. Balaji S, Amadi C, Prasad S, Bala Kasav J, Upadhyay V, Singh AK, et al. Urban rural comparisons of polycystic ovary syndrome burden among adolescent girls in a hospital setting in India. Biomed Res Int. 2015;2015.

2. Joshi B, Mukherjee S, Patil A, Purandare A, Chauhan S, Vaidya R. A cross-sectional study of polycystic ovarian syndrome among adolescent and young girls in Mumbai, India. Indian J Endocrinol. 2014;18(3):317-24.

3. Clayton RN, Ogden V, Hodgkinson J, Worswick L, Rodin DA, Dyer S, et al. How common are polycystic ovaries in normal women and what is their significance for the fertility of the population? Clin Endocrinol. 1992;37(2):127-34.

4. Taylor AE, McCourt B, Martin KA, Anderson EJ, Adams JM, Schoenfeld D, et al. Determinants of Abnormal Gonadotropin Secretion in Clinically Defined Women with Polycystic Ovary Syndrome1. J Clin Endocrinol Metab. 1997;82(7):2248-56.
5. Dutta D. Amenorrhoea. Konar HL, Dutta D, Textbook of Gynecology. 6th ed. Jaypee Brothers; 2013:477.

6. Nahuis MJ, Oosterhuis GJE, Hompes PGA, Van Wely M, Mol BWJ, Van Der Veen F. The basic fertility workup in women with polycystic ovary syndrome: A systematic review. Fertil Steril. 2013;100(1):219-25.

7. Legro RS, Dodson WC, Kris-Etherton PM, Kunselman AR, Stetter CM, Williams NI, et al. Randomized controlled trial of preconception interventions in infertile women with polycystic ovary syndrome. J Clin Endocrinol Metab. 2015;100(11):4048-58.

8. Palomba S, Falbo A, La Sala GB. Metformin and gonadotropins for ovulation induction in patients with polycystic ovary syndrome: A systematic review with meta-analysis of randomized controlled trials. 2014;12.

9. Huber-Buchholz MM, Carey DGP, Norman RJ. Restoration of Reproductive Potential by Lifestyle Modification in Obese Polycystic Ovary Syndrome: Role of Insulin Sensitivity and Luteinizing Hormone1. J Clin Endocrinol Metab. 1999;84(4):1470-4.

10. Al-Azemi M, Omu FE, Omu AE. The effect of obesity on the outcome of infertility management in women with polycystic ovary syndrome. Arch Gynecol Obstet. 2004;270(4):205-10.

11. Robinson S, Kiddy D, Gelding S V., Willis D, Niththyananthan R, Bush A, et al. The relationship of insulin insensitivity to menstrual pattern in women with hyperandrogenism and polycystic ovaries. Clin Endocrinol (Oxf). 1993;39(3):351-5.

12. Nestler JE, Jakubowicz DJ. Decreases in Ovarian Cytochrome P450c17 $\alpha$ Activity and Serum Free Testosterone after Reduction of Insulin Secretion in Polycystic Ovary Syndrome. N Engl J Med. 1996;335(9):617-23.

13. Fleming R, Hopkinson ZE, Michael Wallace A, Greer IA, Sattar N. Ovarian function and metabolic factors in women with oligomenorrhea treated with metformin in a randomized double blind placebocontrolled trial. J Clin Endocrinol Metab. 2002;87(2):569-74.

14. Johnson NP, Stewart AW, Falkiner J, Farquhar CM, Milsom S, Singh VP, et al. PCOSMIC: A multicentre randomized trial in women with PolyCystic Ovary syndrome evaluating metformin for infertility with clomiphene. Hum Reprod. 2010;25(7):1675-83.

15. Morley LC, Tang T, Yasmin E, Norman RJ, Balen AH. Insulin-sensitising drugs (metformin, rosiglitazone, pioglitazone, D-chiro-inositol) for women with polycystic ovary syndrome, oligo amenorrhoea and subfertility. Vol. 2017, Cochrane Database of Systematic Reviews. John Wiley and Sons Ltd; 2017.

16. Lashen H. Review: Role of metformin in the management of polycystic ovary syndrome [Internet]. Vol. 1, Therapeutic Advances in 
Endocrinology and Metabolism. SAGE Publications; 2010;117-28.

17. Meirow D, Laufer N, Schenker JG. Ovulation induction in polycystic ovary syndrome: A review of conservative and new treatment modalities. Eur J Obstet Gynecol Reprod Biol. 1993;50(2):123-31.

18. Brown J, Farquhar C, Beck J, Boothroyd C, Hughes E. Clomiphene and anti-oestrogens for ovulation induction in PCOS [Internet]. Cochrane Database of Systematic Reviews. John Wiley and Sons Ltd; 2009.

19. Kousta E, White DM, Franks S. Modern use of clomiphene citrate in induction of ovulation [Internet]. Vol. 3, Human Reproduction Update. Oxford University Press. 1997;359-65.

20. Balen AH, Morley LC, Misso M, Franks S, Legro $\mathrm{RS}$, Wijeyaratne $\mathrm{CN}$, et al. The management of anovulatory infertility in women with polycystic ovary syndrome: An analysis of the evidence to support the development of global WHO guidance. Hum Reprod Update. 2016;22(6):687-708.

21. Gysler M, March CM, Mishell DR, Bailey EJ. A decade's experience with an individualized clomiphene treatment regimen including its effect on the postcoital test. Fertil Steril. 1982;37(2):161-7.

22. Neveu N, Granger L, St-Michel P, Lavoie HB. Comparison of clomiphene citrate, metformin, or the combination of both for first-line ovulation induction and achievement of pregnancy in 154 women with polycystic ovary syndrome. Fertil Steril. 2007;87(1):113-20.

23. Rossing MA, Daling JR, Weiss NS, Moore DE, Self SG. Ovarian tumors in a cohort of infertile women. N Engl J Med; 1995;8L25-6.

24. Racette L, Casson PR, Claman P, Zackon DH, Casson EJ. An investigation of the visual disturbances experienced by patients on clomiphene citrate. Fertil Steril. 2010;93(4):1169-72.

25. Cole PA, Robinson CH. Mechanism and Inhibition of Cytochrome P-450 Aromatase. J Med Chem. 1990;33(11):2933-42.

26. Lee VCY, Ledger W. Aromatase inhibitors for ovulation induction and ovarian stimulation. Clin Endocrinol. 2011;74:537-46.

27. Franik S, Eltrop SM, Kremer JAM, Kiesel L, Farquhar C. Aromatase inhibitors (letrozole) for subfertile women with polycystic ovary syndrome. Cochrane Databa Systemat Rev. 2018;2018.

28. Rahmani E, Ahmadi S, Motamed N, Maneshi HO. Clinical Study Dosage Optimization for Letrozole Treatment in Clomiphene-Resistant Patients with Polycystic Ovary Syndrome: A Prospective Interventional Study. Obstet Gynecol Int. 2012;2012.

29. Diamond MP, Legro RS, Coutifaris C, Alvero R, Robinson RD, Casson $\mathrm{P}$, et al. Letrozole,
Gonadotropin, or Clomiphene for Unexplained Infertility. N Engl J Med. 2015;373(13):1230-40.

30. Legro RS, Brzyski RG, Diamond MP, Coutifaris C, Schlaff WD, Casson P, et al. Letrozole versus Clomiphene for Infertility in the Polycystic Ovary Syndrome. N Engl J Med. 2014;371(2):119-29.

31. Dale PO, Tanbo T, Lunde O, Åbyholm T. Ovulation induction with low-dose follicle-stimulating hormone in women with the polycystic ovary syndrome. Acta Obstet Gynecol Scand. 1993;72(1):43-6.

32. Fauser BCJM, van Heusden AM. Manipulation of Human Ovarian Function: Physiological Concepts and Clinical Consequences*. Endocr Rev. 1997; 18(1):71-106.

33. Homburg R, Howles CM. Low-dose FSH therapy for anovulatory infertility associated with polycystic ovary syndrome: Rationale, results, reflections and refinements. In: Human Reproduction Update. Hum Reprod Update. 1999;493-9.

34. Tarlatzis BC, Fauser BCJM, Legro RS, Norman RJ, Hoeger K, Pasquali R, et al. Consensus on infertility treatment related to polycystic ovary syndrome. In: Human Reproduction. Oxford University Press. 2008;462-77.

35. Balasch J, Tur R, Peinado JA. The safety and effectiveness of stepwise and low-dose administration of follicle stimulating hormone in WHO group II anovulatory infertile women: Evidence from a large multicenter study in Spain. J Assist Reprod Genet. 1996;13(7):551-6.

36. Kumar P, Sait SF, Sharma A, Kumar M. Ovarian hyperstimulation syndrome. J Hum Reprod Sci. 2011;4:70-5.

37. Tso LO, Costello MF, Albuquerque LE, Andriolo RB, Freitas V. Metformin treatment before and during IVF or ICSI in women with polycystic ovary syndrome. Cochrane Database Syst Rev. 2009.

38. Armar NA, McGarrigle HHG, Honour J, Holownia P, Jacobs HS, Lachelin GCL. Laparoscopic ovarian diathermy in the management of anovulatory infertility in women with polycystic ovaries: Endocrine changes and clinical outcome. Fertil Steril. 1990;53(1):45-9.

39. Agarwal S, Trolice MP. Pregnancy rates after laparoscopic ovarian drilling in polycystic ovary syndrome patients following unsuccessful ovulation induction. Fertil Steril. 2019;112(3):e414.

40. Bordewijk EM, Nahuis M, Costello MF, Van der Veen F, Tso LO, Mol BWJ, et al. Metformin during ovulation induction with gonadotrophins followed by timed intercourse or intrauterine insemination for subfertility associated with polycystic ovary syndrome. Cochra Databa Systemat Rev. 2017;2017.

41. Guzick DS. Ovulation induction management of PCOS. Clin Obstet Gynecol. 2007;50(1):255-67.

Cite this article as: Agrawal S, Das V, Agarwal A, Pandey A. Fetal Doppler for prediction of adverse perinatal outcome in preeclampsia in a low resource setting. Int J Reprod Contracept Obstet Gynecol 2021;10:1236-40. 